\title{
ENHANCED ISOLATED LUNG FUNCTION AFTER ISCHEMIA WITH ANTI-INTERCELLULAR ADHESION MOLECULE ANTIBODY
}

Scott A. Buchanan, MD

Michael C. Mauney, MD

Nuno F. deLima, MD

Oliver A. R. Binns, MD

Jeffrey S. Cope, MD

Kimberly S: Shockey, MS

Susan G. Gordon, MD

Michael B. Erwin, MD

George Sutherland, BA

Irving L. Kron, MD

Curtis G. Tribble, MD
The binding of leukocytes to intercellular adhesion molecules expressed on endothelial surfaces during ischemia and subsequent reperfusion initiates leukocyte-mediated reperfusion injury. Interruption of this leukocyte-endothelium interaction may therefore prevent reperfusion injury. In an isolated, ventilated, blood-perfused rabbit lung preparation, we studied the effect of a monoclonal anti-intercellular adhesion molecule antibody on lung function during reperfusion. Lungs were harvested with $50 \mathrm{ml} / \mathrm{kg}$ cold Euro-Collins flush and $30 \mu \mathrm{g}$ prostaglandin $\mathrm{E}_{1}$ before storage for 18 hours at $4^{\circ} \mathrm{C}$. Experimental groups received low-dose $(100 \mu \mathrm{g})$ or high-dose $(200 \mu \mathrm{g})$ anti-intercellular adhesion molecule antibody added to the pulmonary flush at harvest and to the initial reperfusate. Eighteen-hour control preparations were preserved for 18 hours and received saline solution vehicle. Immediate control preparations were harvested and immediately reperfused. The oxygen tension in the recirculated pulmonary venous effluent was measured after $\mathbf{3 0}$ minutes of reperfusion. Histologic specimens were graded by blinded observers for degree of leukocyte infiltration ( 0 , normal, to 4 , severe infiltration). The mean oxygen tensions ( \pm standard error of the mean) were $138.29 \pm 6.23,58.86 \pm$ 9.14, 86.87 \pm 11.32 , and $139.33 \pm 16.15 \mathrm{~mm} \mathrm{Hg}$ in immediate control preparations, 18-hour control preparations, low-dose antibody group, and high-dose antibody group, respectively $(p=0.0001)$. The leukocyte grades (mean \pm standard error of the mean) were $1.5 \pm 0.723,3.0 \pm 0.955,1.9 \pm$ 0.899 , and $1.2 \pm 0.834$, respectively $(p=0.0002)$. We conclude that antiintercellular adhesion molecule antibody added to the pulmonary flush and initial reperfusate results in a dose-dependent enhancement of the reperfused lung's ability to oxygenate blood, possibly as a result of decreased leukocyte sequestration. (J Thorac Cardiovasc Surg 1996;111:941-7)
$\mathrm{T}$ he sequestration of activated leukocytes within pulmonary allografts has been established as a significant cause of pulmonary reperfusion injury

From the Thoracic and Cardiovascular Research Laboratory, Department of Surgery, University of Virginia Health Sciences Center, Charlottesville, Va.

Funded in part by the National Institutes of Health under RO-1 grant HL 48242 and NRSA fellowship 5 F32 HL 08940. Additional support from $\mathrm{CNPq}$-Conselho Nacional de Desenvolvimento Cientifico e Tecnologico, Brazil, is acknowledged.

Received for publication June 21, 1995; revisions requested Oct. 3, 1995; revisions received Oct. 20, 1995; accepted for publication Dec. 21, 1995.

Address for reprints: Curtis G. Tribble, MD, Professor, Thoracic and Cardiovascular Surgery, Box 310, Department of Surgery, University of Virginia Health Sciences Center, Charlottesville, VA 22908.

Copyright (C) 1996 by Mosby-Year Book, Inc.

$0022-5223 / 96 \$ 5.00+0 \quad \mathbf{1 2 / 6 / 7 1 4 4 5}$ after lung transplantation. ${ }^{1}$ Correspondingly, leukocyte depletion of the recipient by means of filtration methods has been shown to attenuate reperfusion injury in experimental models. ${ }^{2,3}$ Practical difficulties with effective leukocyte filtration and concerns about leukopenia in already immunocompromised patients, however, have prevented the routine clinical application of leukocyte depletion in lung transplant centers. ${ }^{1,4}$ Newer methods of limiting leukocyte-mediated reperfusion injury that exploit our growing understanding of the molecular mechanisms of the leukocyte-endothelium interaction during reperfusion have been proposed. For example, the leukocyte membrane glycoprotein CD18 binds to the endothelium-based intercellular adhesion molecule-1 (ICAM-1), initiating leukocyte demargination early in reperfusion. ${ }^{5,6}$ Monoclonal antibodies have been developed that bind to the ICAM-1 receptor and prevent this interaction. ${ }^{7-9}$ 
We hypothesized that the administration of an anti-ICAM-1 monoclonal antibody to pulmonary allografts before reperfusion would decrease leukocyte sequestration and thus attenuate reperfusion injury.

\section{Materials and methods}

We developed a model of isolated, ventilated, and blood-perfused rabbit lung function, which was employed in this protocol. All animals received humane care in compliance with the "Principles of Laboratory Animal Care" formulated by the National Society for Medical Research and the "Guide for the Care and Use of Laboratory Animals" prepared by the Institute of Laboratory Animal Resources and published by the National Institute of Health (NIH Publication No. 86-23, revised 1985).

Harvest procedure. Forty New Zealand White rabbits weighing 3.0 to $3.5 \mathrm{~kg}$ were used as lung donors. Each animal was anesthetized with intramuscular ketamine (50 $\mathrm{mg} / \mathrm{kg}$ ) and xylazine $(5 \mathrm{mg} / \mathrm{kg})$. A tracheostomy was performed and mechanical ventilation was instituted with a constant-pressure ventilator (RSP1002; Kent Scientific Corporation, Litchfield, Conn.) on room air and a rate of 20 breaths/min. A median sternotomy was then performed and thymectomy was carried out. The two superior venae cavae and one inferior vena cava were loosely encircled with ligatures, and the pericardium was opened. Both the pulmonary artery (PA) and the aorta were dissected free and similarly encircled. A purse-string suture was then placed in the free wall of the right ventricle, and the rabbit was heparinized $(500 \mathrm{U} / \mathrm{kg})$. After injection of $30 \mu \mathrm{g}$ prostaglandin $\mathrm{E}_{1}$ into the $\mathrm{PA}$, the venae cavae were interrupted and the onset of ischemia was noted. The PA was then cannulated through the right ventricular pursestring, and both the right ventricular and PA ligatures were tied around the cannula. After venting of the left ventricle and ligation of the aorta, $50 \mathrm{mg} / \mathrm{kg}$ of saline solution at $4^{\circ} \mathrm{C}$ was infused into the PA from a height of $30 \mathrm{~cm}$. Topical cooling was achieved with cold saline solution slush. During PA flush, the left atrium was cannulated through a left ventricular purse-string. After completion of the PA flush, the inflow and outflow cannulas were clamped. Care was taken to leave the pleurae intact until the completion of the flush to avoid parenchymal injury. The tracheostomy tube was then clamped at end-inspiration and the heart-lung block was excised. The lungs were stored at $4^{\circ} \mathrm{C}$ in saline solution.

Reperfusion procedure. Forty-five heparinized and anesthetized New Zealand White rabbits served as venous blood donors. Saline solution was added to each aliquot of blood to achieve a hematocrit of $28 \%$ to $30 \%$. During reperfusion, the lungs were suspended by a force transducer in a warmed, humidified chamber. Room air ventilation at 20 breaths/min was reestablished with a constantpressure ventilator. The initial maximum inspiratory pressure was set to achieve a tidal volume of $10 \mathrm{ml} / \mathrm{kg}$ of donor rabbit weight and was subsequently held constant. Any lung with evidence of air leakage was excluded. The inflow and outflow cannulas were then connected to a venous blood-filled perfusion circuit, with care taken to avoid the introduction of air bubbles. The circuit (Kent Scientific Corporation) was designed to recirculate $200 \mathrm{ml}$ warmed blood with a roller pump (7521-40; Cole-Parmer Instrument Company, Niles, Ill.) and a blood filter (2C7600; Baxter Healthcare Corp., Deerfield, Ill.) at a rate of $40 \mathrm{ml} / \mathrm{min}$. Continuous recording of PA pressure, pulmonary venous pressure, lung weight, airway flow, and airway pressure was carried out with a dynamic dataacquisition program (Workbench PC; Strawberry Tree, Inc., Sunnydale, Calif.) run on a personal computer (470A; Compaq Prolinea, Houston, Texas). This program allowed immediate calculation of tidal volume, pulmonary vascular resistance (Pulmonary vascular resistance $=80 \times$ [PA pressure - Pulmonary venous pressure]/Flow), and dynamic airway compliance (Dynamic airway compliance $=$ Tidal volume/Airway pressure). The pulmonary venous pressure was maintained between 5 and $8 \mathrm{~mm} \mathrm{Hg}$ by changing the height of an outflow reservoir in the circuit. Pulmonary venous blood samples were collected for blood gas analysis (Corning $178 \mathrm{pH} / \mathrm{Blood}$ Gas Analyzer; Corning Inc., Corning, N.Y.) at 1, 10, 20, and 30 minutes after the start of reperfusion. Oxygen contact with exposed blood surfaces inside reservoir containers was minimized by the continuous passive infusion of $100 \%$ nitrogen.

Experimental protocol. Ten double-lung blocks were reperfused immediately after harvest, serving as immediate control (IC) preparations. For our ischemic groups, we chose a storage interval of 18 hours, after a series of preliminary experiments in which 24 hours of cold storage was associated with a rapid hemorrhiagic demise of the lung before completion of 30 minutes of reperfusion. By contrast, 6- and 12-hour ischemic intervals produced little measurable lung injury. Ten lungs were stored for 18 hours at $4^{\circ} \mathrm{C}$ and then reperfused, serving as 18 -hour control preparations. Two treatment groups were similarly harvested and stored at $4^{\circ} \mathrm{C}$ for 18 hours but received low- or high-dose antibody therapy, respectively. Ten lungs in the low-dose group received $100 \mu \mathrm{g}$ purified anti-ICAM-1 monoclonal antibody (RR1; Boehringer Ingelheim Corp., Ridgefield, Conn.) administered intravascularly with the last $20 \mathrm{ml}$ of Euro-Collins flush during harvest and a second $100 \mu \mathrm{g}$ dose intravascularly in the initial reperfusate. Ten lungs in the high-dose group received $200 \mu \mathrm{g}$ anti-ICAM-1 antibody administered in a similar manner, both during harvest and again before reperfusion. All lungs were reperfused for 30 minutes.

At the completion of the study, samples of the left lower lobe were weighed and dried for calculation of wet-to-dry weight ratios. Histologic specimens were taken from the right lower lobe and placed in formalin. The histologic specimens were graded for the degree of leukocyte sequestration by three blinded observers according to a 5 -point scale modified from the method of Yousem and coworkers. ${ }^{10}$ The grading classification is outlined in Table I.

Statistical analysis. Statistical analysis was performed for the four groups by means of analysis of variance on the software STATISTICA (StatSoft, Inc., Tulsa, Okla.). Significant differences were determined by means of Tukey's Honestly Significant Difference test. The data are reported as mean \pm standard error of the mean. 
Table I. Grading of leukocyte sequestration

\begin{tabular}{cl}
\hline Grade & \multicolumn{1}{c}{ Description } \\
\hline 0 & No evidence of leukocyte sequestration; normal lung \\
1 & Less than 25\% of interstitium infiltrated by leukocytes \\
2 & $50 \%$ of interstitium infiltrated by leukocytes \\
3 & $75 \%$ of interstitium infiltrated by leukocytes \\
4 & $100 \%$ of interstitium infiltrated by leukocytes \\
\hline
\end{tabular}

\section{Results}

The capacity of the room air-ventilated lungs to oxygenate blood at the completion of the 30-minute period of reperfusion revealed an expected impairment in 18-hour control lungs compared with the IC group. Administration of anti-ICAM-1 monoclonal antibody markedly enhanced the oxygenation capacity in a dose-dependent manner (Fig. 1). By contrast to the IC and 18-hour control groups, the lungs receiving low- and high-dose anti-ICAM-1 antibody were capable of generating oxygen tensions of $86.87 \pm 11.32$ and $139.33 \pm 16.15$, respectively $(p=$ 0.0001 ). The degree of leukocyte sequestration was also increased in the 18-hour control group compared with the IC group. Administration of antiICAM-1 antibody was associated with decreased histologic evidence of leukocyte sequestration, again in a dose-dependent manner (Fig. 2). The leukocyte grade (mean \pm standard error of the mean) was 1.5 \pm 0.723 in the IC group versus $3.0 \pm 0.955$ in the 18 hour-stored lung group. In lungs receiving antibody, the mean leukocyte grade was improved at $1.9 \pm$ 0.899 in the low-dose group and $1.2 \pm 0.834$ in the high-dose group $(p=0.0002)$. Representative histologic specimens taken from each cohort of reperfused lungs revealed a marked reduction in leukocyte sequestration associated with the administration of blocking antibody (Fig. 3). Measures of PA pressure, pulmonary vascular resistance, airway compliance, lung weight, and wet-to-dry weight ratios were not improved by the administration of anti-ICAM-1 antibody. These data are presented in Table II.

\section{Discussion}

The complex molecular events that orchestrate adhesion between leukocytes and vascular endothelium during inflammatory responses have been well characterized. ${ }^{5,6}$ Leukocyte adhesion to endothelial cells is mediated by a group of glycoproteins expressed on the surface of leukocytes known as $\beta_{2}$ integrins. Each integrin is comprised of a common $\beta$-subunit (CD18) bound to one of $3 \alpha$-subunits (CD11a, CD11b, and CD11c) forming three integrin

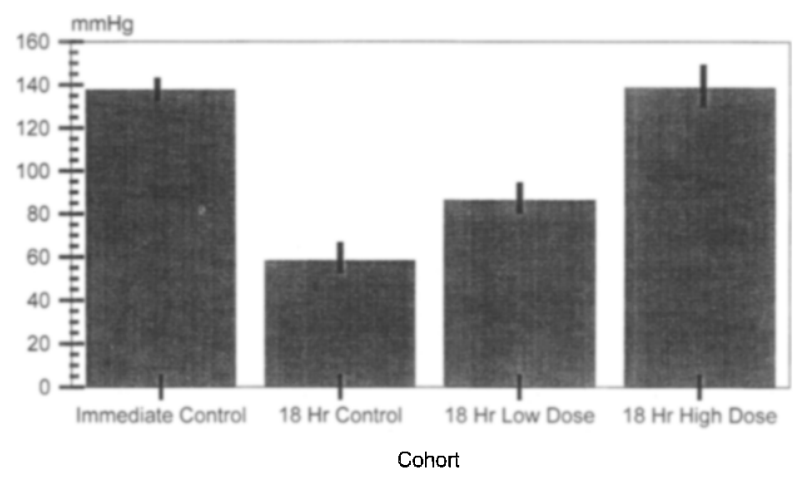

Fig. 1. Oxygenation capacity (partial pressure of oxygen in millimeters of mercury) after 30 minutes of reperfusion. $p=0.0001$ for 18-hour control group versus low-dose group, 18-hour control group versus high-dose group, low-dose versus high-dose group, and IC group versus 18-hour control group.

heterodimers: CD11a/CD18, CD11b/CD18, and $\mathrm{CD} 11 \mathrm{c} / \mathrm{CD} 18{ }^{7}$ The CD11a/CD18 and CD11b/CD18 molecules bind to an endothelium-based receptor called ICAM-1, initiating the process of leukocyte demargination. Various proinflammatory mediators such as interleukin-1 and tumor necrosis factor released in response to ischemia both increase the expression of these adhesion molecules and enhance their binding effectiveness. ${ }^{8,11}$ Reperfusion of the ischemic pulmonary vasculature with whole blood is therefore associated with a profound sequestration of leukocytes within the allograft. Subsequent allograft damage caused by leukocyte-generated reactive oxygen species and proteolytic enzymes ${ }^{11-14} \mathrm{can}$ result in acute lung dysfunction after transplantation.

Because the adhesion of activated leukocytes to damaged endothelium is thought to be an early and pivotal event in the pathophysiology of reperfusion injury after ischemia, a number of investigators have tried to reduce solid organ reperfusion injury by blocking this initial leukocyte-endothelium interaction. Antibodies directed against the leukocyte CD18 complex (anti-CD18) ${ }^{15}$ and against the endothelial ICAM-1 receptor (anti-ICAM-1) ${ }^{16}$ have been shown to minimize neurologic deficits during reperfusion after ischemia of the rabbit central nervous system. Anti-ICAM-1 therapy has been shown to be protective in a model of kidney ischemia-reperfusion. ${ }^{17}$ Leukocyte antiadhesion therapy also appears to limit the size of myocardial infarction, ${ }^{18}$ minimize the severity of systemic inflammation after cardiopulmonary bypass, ${ }^{19,20}$ and prevent 


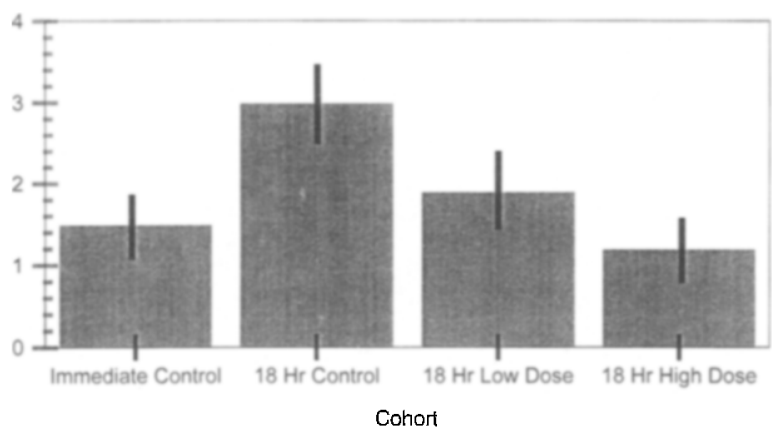

Fig. 2. Degree of leukocyte sequestration after $30 \mathrm{~min}-$ utes of reperfusion. $p=0.0002$ for 18 -hour control group versus low-dose group, 18-hour control group versus high-dose group, and IC group versus 18-hour control group.

myocardial stunning, low reflow, and edema formation after experimental heart transplantation. ${ }^{21}$

Evidence that preventing leukocyte adhesion within the pulmonary capillary bed protects the lung from the effects of ischemia and reperfusion has also been reported. Schueler and coworkers ${ }^{3}$ demonstrated that leukocyte depletion in a porcine model of double-lung transplantation improves the ischemic tolerance of the lung, with excellent lung function noted after 24 hours of preservation. Kapelanski and colleagues ${ }^{7}$ reported that an antiCD18 monoclonal antibody was capable of improving gas exchange and decreasing lung water accumulation after 4 hours of preservation and 6 hours of reperfusion in a model of canine single-lung transplantation. Interestingly, other features of lung reperfusion injury, including pulmonary hypertension, increased pulmonary vascular resistance, and reduced airway compliance, were not improved with the antibody. Horgan and colleagues ${ }^{8,9}$ carried out experiments in which 24 hours of in situ rabbit lung ischemia was followed by 2 hours of reperfusion. They showed, with antibodies directed against both CD18 and ICAM-1, that neutrophil sequestration within the pulmonary vascular microcirculation is enhanced by upregulation of the ICAM-1 receptor during ischemia and is a major determinant of lung reperfusion injury. Measures of oxygenation capacity, pulmonary vascular hemodynamics, and airway compliance during reperfusion in these experiments were not recorded.

To further evaluate the role of leukocytes in mediating lung reperfusion injury and to determine whether antagonism of the ICAM-1 receptor could ameliorate the effects of this injury, we employed an isolated, ventilated, blood-perfused model of rabbit lung function with which we have experience. With RR1, a monoclonal antiICAM-1 antibody, we were able to demonstrate a dose-dependent enhancement in rabbit lung oxygenation capacity after 18 hours of cold preservation and 30 minutes of reperfusion. This improved oxygenation correlated with decreased leukocyte sequestration. Similar to the results of Kapelanski and associates, ${ }^{7}$ we were unable to achieve improvements in reperfusion-associated pulmonary hypertension or reduced airway compliance. These features of reperfusion injury in the lung may be mediated either wholly or in part by leukocyte-independent or ICAM-1-independent mechanisms.

All of the lungs subjected to 18 hours of ischemia, regardless of anti-ICAM-1 therapy, accumulated lung water, as measured by increased lung weight during reperfusion and by increased wet-to-dry weight ratios at the completion of reperfusion. These findings suggest that the frequently observed capillary leakage phenomenon can occur without significant infiltration of leukocytes in this model and that measures designed to prevent leakage may need to be more broadly focused than any specific antileukocyte therapy. Because the administration of an isotype-matched control monoclonal antibody directed against an adhesion-independent leukocyte antigenic determinant was not used, we cannot exclude the remote possibility that nonspecific effects of monoclonal antibody administration contributed to our results.

Although the safety and efficacy of anti-ICAM-1 monoclonal antibody in human beings has yet to be firmly established, a phase I trial of its use as an immunosuppressant in renal allograft recipients has found no significant adverse side effects. ${ }^{22} \mathrm{~A}$ second published trial of anti-ICAM-1 therapy as an antiinflammatory agent in patients with rheumatoid arthritis has further substantiated the drug's safety, with reported clinical improvement lasting as long as 55 days after completion of a 5-day course of anti-ICAM-1 therapy. ${ }^{23}$

In conclusion, the administration of a monoclonal anti-ICAM-1 antibody prevented the impairment of oxygenation capacity and was capable of reducing leukocyte infiltration in this model of isolated lung function. These data provide additional evidence of the detrimental effect of leukocytes during pulmonary reperfusion after ischemia. Further, these data 

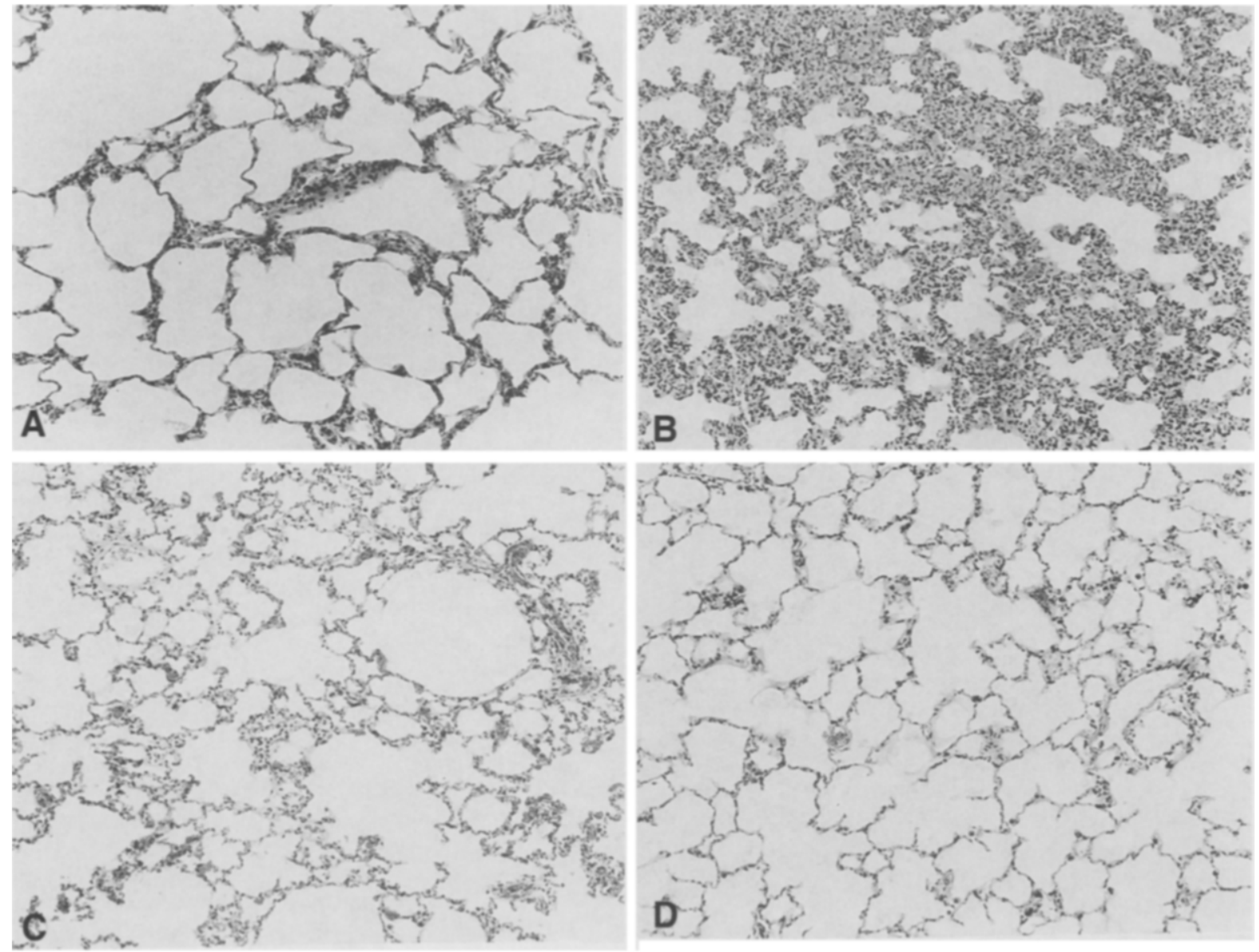

Fig. 3. Histologic specimens (Hematoxylin-eosin stain; original magnification $\times 125$ ). A, Reperfused immediately after harvest (IC group). Minimal interstitial infiltration with inflammatory cells. Normal architecture preserved. B, Reperfused after 18 hours of cold ischemia (18-hour control group). Severe infiltration with polymorphonuclear cells and erythrocytes with distortion of the microscopic architecture. C, Low-dose antibody and 18 hours of cold ischemia (low-dose group). Moderate infiltrate with polymorphonuclear cells and well-preserved architecture. D, High-dose antibody and 18 hours of cold ischemia (high-dose group). No interstitial infiltrate with inflammatory cells. Architecture well preserved.

Table II. Parameters unaffected by administration of anti-ICAM-1 antibody

\begin{tabular}{lccccc}
\hline & $\begin{array}{c}\text { PA pressure } \\
(\mathrm{mm} \mathrm{Hg})\end{array}$ & $\begin{array}{c}P V R \\
\left(\text { dynes } \cdot \text { sec } / \mathrm{cm}^{-7}\right)\end{array}$ & $\begin{array}{c}\text { Airway compliance } \\
(\% \text { change })\end{array}$ & $\begin{array}{c}\text { Lung weight } \\
\text { (\% change) }\end{array}$ & $\begin{array}{c}\text { Wet-to-dry } \\
\text { weight ratio }\end{array}$ \\
\hline IC & $21.37 \pm 1.91$ & $55.18 \pm 5.45$ & $3.92 \pm 8.26$ & $15.93 \pm 8.27$ & $4.65 \pm 0.72$ \\
18-hour control & $46.69 \pm 9.20$ & $87.90 \pm 18.37$ & $-26.73 \pm 7.28$ & $103.13 \pm 29.92$ & $7.30 \pm 0.69$ \\
18-hour low-dose group & $43.34 \pm 7.38$ & $82.75 \pm 14.47$ & $-39.04 \pm 5.89$ & $136.67 \pm 26.95$ & $6.74 \pm 0.95$ \\
18-hour high-dose group & $36.26 \pm 4.63$ & $69.66 \pm 9.54$ & $-23.74 \pm 8.41$ & $93.18 \pm 17.90$ & $7.79 \pm 1.21$ \\
$p$ & $0.0396 ;$ IC vs & NS & $0.0024 ;$ IC vs & $0.0044 ;$ IC vs & NS \\
& 18-hour control & & 18-hour control & 18-hour control \\
& & & and IC vs 18-hour & and IC vs 18-hour \\
& & & low-dose group & low-dose group \\
\hline
\end{tabular}

Data listed represent mean values \pm standard error of the mean recorded at the end of a 30 -minute period of reperfusion. PVR, Pulmonary vascular resistance; $N S$, not significant. 
support the importance of the endothelial ICAM-1 receptor in mediating leukocyte sequestration in reperfusion-injured lungs.

We express our appreciation to Mr. Anthony Herring for his invaluable technical assistance.

\section{REFERENCES}

1. Novick RJ, Menkis AH, McKenzie FN. New trends in lung preservation: a collective review. J Heart Lung Transplant 1992;11:377-92.

2. Bando K, Pillai R, Cameron DE, et al. Leukocyte depletion ameliorates free radical-mediated lung injury after cardiopulmonary bypass. J Thorac Cardiovase Surg 1990;99:873-77.

3. Schueler S, De Valeria PA, Hatanaka M, et al. Successful twenty-four-hour lung preservation with donor core cooling and leukocyte depletion in an orthotopic lung transplantation model. J Thorac Cardiovasc Surg 1992;104:73-82.

4. Bando K, Schueler S, Cameron DE, et al. Twelve-hour cardiopulmonary preservation using donor core cooling, leukocyte depletion, and liposomal superoxide dismutase. J Heart Lung Transplant 1991;10:304-9.

5. Springer TA. Adhesion receptors of the immune system. Nature 1990;346:425-34.

6. Lefer AM, Lefer DJ. Pharmacology of the endothelium in ischemia-reperfusion and circulatory shock. Annu Rev Pharmacol Toxicol 1993;33:71-90.

7. Kapelanski DP, Iguchi A, Niles SD, Mao HZ. Lung reperfusion injury is reduced by inhibiting a CD18-dependent mechanism. J Heart Lung Transplant 1993;12:294-307.

8. Horgan MJ, Ge M, Gu J, Rothlein R, Malik AB. Role of ICAM-1 in neutrophil-mediated lung vascular injury after occlusion and reperfusion. Am J Physiol 1991;261:H1578-84.

9. Horgan MJ, Wright SD, Malik AB. Antibody against leukocyte integrin (CD18) prevents reperfusion-induced lung vascular injury. Am J Physiol 1990;259:L315-9.

10. Yousem SA, Berry GJ, Brunt EM, et al. A working formulation for the standardization of nomenclature in the diagnosis of heart and lung rejection: lung rejection study group. J Heart Transplant 1990;9:593-601.

11. Lucchesi BR. Modulation of leukocyte-mediated myocardial reperfusion injury. Annu Rev Physiol 1990;52:561-76.

12. Weiss SJ. Tissue destruction by neutrophils. N Engl J Med 1989;320:365-76.

13. Dickstein RA. Reperfusion injury: the role of leukocytes. Perfusion Life 1990;7:34-78.

14. Gorlick DL, Ortolano GA. Leukocyte depletion and implications for the prevention of reperfusion injury. Proc Am Acad Cardiovasc Perfusion 1992;13:154-65.

15. Clark WM, Madden KP, Rothlein R, Zivin JA. Reduction of central nervous system ischemic injury in rabbits using leukocyte adhesion antibody treatment. Stroke 1991;22:877-83.

16. Bowes MP, Zivin JA, Rothlein R. Monoclonal antibody to the ICAM-1 adhesion site reduces neurological damage in a rabbit cerebral embolism stroke model. Exp Neurol 1993; 119:215-9.

17. Kelly KJ, Williams WW, Colvin RB, Bonventre JV. Antibody to intercellular adhesion molecule 1 protects the kidney against ischemic injury. Proc Natl Acad Sci U S A 1994;91: 812-6.

18. Simpson PJ, Todd RF, Fantone JC, Mickelson JK, Griffin JD,
Lucchesi BR. Reduction of experimental canine myocardial reperfusion injury by a monoclonal antibody (anti-MO1, anti-CD11b) that inhibits leukocyte adhesion. J Clin Invest 1988:81:624-9.

19. Verrier ED, Shen I. Potential role of neutrophil anti-adhesion therapy in myocardial stunning, myocardial infarction, and organ dysfunction after cardiopulmonary bypass. J Card Surg 1993;8(Suppl):309-12.

20. Gillinov AM, Redmond JM, Zehr KJ, et al. Inhibition of neutrophil adhesion during cardiopulmonary bypass. Ann Thorac Surg 1994;57:126-33.

21. Byme JG, Smith WJ, Murphy MP, Couper GS, Appleyard $\mathrm{RF}$, Cohn LH. Complete prevention of myocardial stunning, contracture, low-reflow, and edema after heart transplantation by blocking neutrophil adhesion molecules during reperfusion. J Thorac Cardiovasc Surg 1992;104:1589-96.

22. Haug CE, Colvin RB, Delmonico FL, et al. A phase I trial of immunosuppression with anti ICAM-1 (CD54) mAb in renal allograft recipients. Transplantation 1993;55:766-73.

23. Kavanaugh AF, Davis LS, Nichols LA, et al. Treatment of refractory rheumatoid arthritis with a monoclonal antibody to intercellular adhesion molecule 1. Arthritis Rheumatism 1994;37:992-9.

\section{Discussion}

Dr. Margaret D. Allen (Seattle, Wash.). This is certainly an elegant study in an ex vivo model of clinical reperfusion injury after lung transplantation, which certainly is a major clinical problem. I appreciate that this is a very difficult and sophisticated model. I do have a few questions.

First, as you know, many lung operations do not require bypass, and bypass circuitry in your experiment itself could potentially result in upregulation of cytokines, adhesion molecules, and other factors. To what extent could this have influenced your results, either pro or con?

Dr. Buchanan. There is plastic tubing to which the blood is exposed in our circuit. This blood is passed through a filter. Our leukocytes are undoubtedly activated, and our adhesion receptors are potentially upregulated. Our system-like any isolated experimental preparation-may introduce some other variables.

Dr. Allen. Second, the monoclonal antibody RR1 that you used binds only to the first and second domains of ICAM-1 and thus blocks the LFA-1 binding site but not the Mac-1 binding site, which is the most important for neutrophils. There are other anti-ICAM-1 antibodies that do block both. Could this possibly explain why you saw improvement in some parameters but not in others? Did you look at what leukocyte subpopulations were present? For instance, were macrophages present, or were you selecting out certain populations?

Dr. Buchanan. The RR1 antibody we used is known to be extremely specific, and in fact I believe this explains our lack of improvement in hemodynamics and airway function. I think you are right. If we looked at subpopulations of cells involved or the particular type of ICAM receptor involved, we would see that only a subset was blocked by our experiment.

Dr. Allen. Finally, I noticed that you did not use an isotype-matched antibody in your control preparations, and I was just wondering about your thoughts on this. 
Dr. Buchanan. I am not convinced of the utility of isotype-matched antibody in this preparation.

Dr. Allen. It would be a nonfunctional antibody to control for binding to $\mathrm{FC}$ receptors.

Dr. Buchanan. We did not use such an antibody in this experiment, but perhaps it would be interesting to do so in the future.

Dr. Allen. I think that the approach you describe would certainly have potential for clinical application.

Dr. Alden H. Harken (Denver, Colo.). I compliment you for initiating a discussion that $I$ think is going to be a theme of today's program. We have several papers on the program that allude to the fact that all cardiac surgeons place patients on cardiopulmonary bypass, and if we leave them there for an hour we can culture bacteria from our cardiopulmonary bypass solution. Whether or not we like to acknowledge that, we are then elaborating or provoking endotoxemia, which then may elaborate tumor necrosis factor, with or without complement, and then express the CD-11 and CD-18 neutrophil receptors and upregulate the ICAM on our endothelial cells, blowing holes in endothelial cells and transudating fluid and albumin into the lungs. Whether we acknowledge that or not, it happens in all of our patients, sometimes subclinically and sometimes not. I have two interrelated questions. One is related to your model. As I look at your data, and I agree with your interpretation completely, in your high- and low-dose antibody systems I wanted to see a relationship between the adherence of neutrophils to those endothelial cells and oxygenation. I saw no difference with the highand low-dose antibody relative to neutrophils but I did see a significant improvement in oxygenation, and that relates to my second question. If you can break this sequence, which you and I both want to do, why have all the clinical studies looking at multiple organ failure or whatever parameter you want against antiendotoxin, anti-tumor necrosis factor, or anti-ICAM-1 been failures? I would like your interpretation of these observations.

Dr. Buchanan. I will approach the second observation first. I suspect the problems that we are seeing after bypass in endotoxic shock are multifactorial, and each of the interventions- the use of an antibody to ICAM, the use of an antibody to endotoxin-by contrast is extremely specific. Perhaps we need to combine these modalities to see some more clinically relevant response.

Regarding your first point, I think that one of the important techniques we need to develop in the laboratory, and we are in the process of doing just that, is figuring out a way to actually look at the leukocyte binding to the endothelium, for example by means of an immunohistochemical technique. It would be interesting to see the exact numbers of leukocytes that are binding, where they are binding, and if in fact they are binding to the ICAM-1 receptor. We really do not know that.

Dr. David A. Fullerton (Denver, Colo.). I too congratulate you on a very pretty study, and I also think it is clinically relevant, which adds to its value. I have a couple of questions.

First, our interest has been in the vascular component of reperfusion injury, and I was curious as to whether you had the opportunity to discern whether your antibody might preserve vascular function after reperfusion.

Dr. Buchanan. We did not look at that in this particular experiment. It would certainly be interesting, for example, to take rings from our lungs and put them on a constriction apparatus to see whether the ability for endotheliumdependent relaxation was preserved. We are in the process of carrying out some such experiments now.

Dr. Fullerton. In addition, 18 hours of ischemia is of course a fairly large insult, and I was curious as to whether you have tried shorter periods of ischemia. If so, are those results substantially different from these, or did you choose 18 hours for some methodologic reason?

Dr. Buchanan. Preliminary data suggested that these rabbit lungs are quite sensitive to ischemia, with a 24-hour period of ischemia resulting in many of the lungs simply crashing and not surviving even the 30 minute period of reperfusion. By contrast, after just 2 or 4 hours of ischemia, lungs studied reveal little evidence of reperfusion injury. In an effort to unmask a significant but treatable injury, we settled on 18 hours.

Dr. Fullerton. Finally, is it necessary to provide the antibody both at the end of your flush and on reperfusion, or is it sufficient to provide it simply on reperfusion?

Dr. Buchanan. I cannot answer that from our data, but I would guess that if I had to administer antibody at one point or the other I would choose to do so just before reperfusion. Theoretically, these ICAM receptors are not significantly upregulated immediately after harvest or during the flush. Time is required for messenger RNA to be produced and translated to actually result in upregulation of the receptor. 